\title{
EL ENFOQUE DE SRAFFA Y LA \\ HISTORIA ECONOMICA
}

\author{
A. BARCELO \\ Universidad de Barcelona
}

El pasado verano murió en Cambridge (Inglaterra), recién cumplidos los ochenta y cinco años de edad, Piero Sraffa, uno de los grandes economistas del siglo xx. Su aportación fundamental se halla condensada en un pequeño libro titulado Producción de mercancias por medio de mercancías. Un objetivo central de esta obra era proponer los cimientos sobre los que edificar un esquema teórico contrapuesto al que ha dominado el panorama de la economía pura después de la contrarrevolución marginalista. La crítica constructiva estribaba en mostrar que los «precios de producción» (parientes cercanos de los prix nécessaire, natural price y valor de los economistas clásicos) constituyen una propiedad emergente de los sistemas económicos reproductivos que puede ser cuantificada atendiendo exclusivamente a las interdependencias productivas. De la base conceptual estaban ausentes, por lo tanto, nociones tales como "producto marginal», "coste marginal» o «utilidad marginal», cuya utilización suele presuponer que la configuración de un sistema económico se halla determinada por pequeñas variaciones reales o virtuales de curvas de oferta y de demanda.

A raíz de la publicación de esta obra (1960), las reacciones fueron muy dispares, formando un amplio abanico que incluía desde la irritación y el menosprecio hasta el entusiasmo, pasando por la perplejidad. No faltaban razones para cualquiera de estas respuestas. Con una rápida ojeada, uno se percata de la extraña originalidad y estilo un tanto hermético en que se presenta el libro. La obra empezó a gestarse a finales de los años veinte. Los únicos economistas mencionados son Quesnay, Smith, Malthus, Ricardo, Torrens, Marx, Marshall, Wicksteed y Keynes. El libro más reciente al que se hace referencia expresa es Theorien über den Mebrwert. La evidentísima preocupación por la exactitud y el rigor no se traducen en un tratamiento formalizado, sino en argumentaciones presentadas en forma de razonamiento cuidadoso sin remitir a teoremas matemáticos (aunque. a menudo estén pre- 
sentes y ocultos). Los temas tratados forman un vasto territorio: el primero de los doce capítulos se ocupa de la «Producción de subsistencia», y el último, de "Desplazamientos en los métodos de producción», con capítulos intermedios sobre "La mercancía patrón», «Reducción a cantidades de trabajo fechadas», «Capital fijo» o «Tierra». El subtítulo reza: «Preludio a una crítica de la teoría económica» (la versión italiana, revisada por el autor, dice "premisas» en lugar de «preludio»). En el prefacio, en fin, se afirma que es «un rasgo peculiar del conjunto de proposiciones ahora publicadas que, aunque no entran en una discusión de la teoría marginalista del valor y de la distribución, han sido elaboradas, sin embargo, para servir de base a una crítica de tal teoría».

El paso de los años, como ocurre para con ciertos vinos, ha ido valorizando esta obra de Sraffa, que se ha convertido ya en un texto clásico. Porque, más allá de las rarezas, extravagancias y homenajes crípticos del autor, contenía una profunda reflexión sobre la naturaleza de los sistemas económicos. En este sentido, es preciso resaltar que una nota característica y distintiva del enfoque sraffiano es la concepción sistémica de las actividades económicas, o sea, la contemplación de la economía como un conjunto de procesos transformadores vinculados por los requerimientos reproductivos. En el marco de esta visión, el objetivo de Sraffa se concreta en analizar las relaciones estructurales básicas de un sistema capaz de autorreproducirse. En ciertos contextos (o bajo supuestos no descaradamente irreales) se muestra que las relaciones de producción y de consumo productivo son condiciones necesarias y suficientes para la determinación de propiedades económicas primordiales.

Para lograr esos objetivos, Sraffa se apoyó esencialmente en la tradición teórica ricardiano-marxista, a la que incorporó algunos avances destacados del análisis económico contemporáneo, singularmente ciertos procedimientos walrasianos de representación desagregada e interdependiente. Un mérito destacado estriba en que consiguió la superación de muchos obstáculos con los que había topado aquella corriente de pensamiento, con lo que ponía de manifiesto a la vez la modernidad de sus ideas nucleares y la necesidad de una decidida revisión conceptual. La consiguiente «revolución cultural» no resultó agradable ni para la economía académica, cuyas bases eran vigorosamente zarandeadas, ni para el epigonismo marxista, más proclive a conservar un legado teórico embalsamado que a utilizarlo como plataforma corregible.

\section{Resultados}

Presentamos a continuación un esbozo de los principales resultados obtenidos por Sraffa. Conviene advertir, de entrada, que se examinan economías 
totalmente desagregadas y en estado estacionario. Los datos del problema son, pues, cantidades físicas de diversos bienes que se transforman al cabo de un tiempo en cantidades físicas de otros bienes. Las tablas así expuestas pueden interpretarse como «fotografías» de los procesos productivos. Las incógnitas son los precios y las variables distributivas (por orden de aparición: tipo de beneficios, salario, renta de la tierra).

En primer lugar, se demuestra que en un sistema de subsistencia en el que los bienes son producidos por diferentes «industrias» (o «procesos») hay un único conjunto de valores de cambio que, de ser adoptado, restablece la situación de partida y permite la reproducción del sistema. En otras palabras, es inmediato el paso de la tabla de transformaciones al sistema de ecuaciones resultante de expresar los procesos en el ámbito homogeneizador de la valoración económica. Queda así probado que los valores de cambio surgen directamente de los métodos de producción, si se sobreentiende un mínimo de estabilidad y automatismo.

A continuación se abordan los problemas derivados de la aparición de un excedente, así como la dilucidación de este concepto clave. El excedente es concebido como una propiedad del sistema económico y no de los procesos productivos individuales. Se define sencillamente como productos menos insumos, y ha de ser representado mediante un vector, dado que los bienes son heterogéneos. El fenómeno del excedente impone la introducción de variables distributivas que expresen la forma en que se distribuye. Se complica entonces la determinación de los precios, que se hallan afectados por las pautas distributivas. Suponiendo conocido el salario real, las ecuaciones de producción permiten determinar, sin embargo, los precios y el tipo de beneficios.

Luego se introduce el salario como variable distributiva adicional y se demuestra que, «incluso si los trabajadores pudieran vivir del aire» (Marx), el tipo de beneficios correspondiente a un sistema dado tiene un valor máximo («tipo máximo de beneficios»), simbolizado por $R$. Mediante algunos artificios matemáticos se llega a la ecuación fundamental:

$$
r=R(1-w)
$$

en la que $r$ significa el tipo de beneficios por período («añow), y $w$, la proporción de los salarios sobre la renta nacional patrón. La renta nacional patrón es un concepto que se apoya en el hecho de que a todo sistema efectivo se le puede asociar un sistema ideal que permite obtener una mercancía compuesta (la «renta nacional patrón») que actúa como patrón de valor invariable ante cambios en la distribución. De este modo se alcanza una ecuación sencilla que condensa la contraposición de intereses entre capital y trabajo asalariado. 
Vale la pena señalar también que la distinción clásica entre bienes de primera necesidad y bienes de lujo es retomada y perfilada por Sraffa, que la reemplaza por la de bienes básicos y no básicos. Los primeros son aquellos directa $o$ indirectamente necesarios para producir todas las mercancías, mientras que los segundos no intervienen como insumos en la fabricación de las restantes mercancías. La situación estratégica de unos y otros es, pues, distinta: sólo los básicos tienen un papel central en la determinación del tipo de beneficios. Más aún, para los no básicos la óptica de los «costes de producción» resulta adecuada, pero con los básicos es impropia debido a que se hallan situados en una trama de interdependencias en la que son fines y medios.

Conviene reseñar, asimismo, un aspecto relativo al tratamiento del capital fijo. Sraffa sigue la regla de Torrens-Von Neumann, consistente en analizar el capital fijo como producción conjunta, esto es, en concebir que el producto de un proceso en el que intervienen medios de producción que operan como totalidades y no se incorporan al bien final, sino que se van desgastando con el uso, se compone conjuntamente de dicho bien final y del medio de producción envejecido (paño y máquina, leche y vaca). Aunque esta manera de ver las cosas pueda parecer algo retorcida, concuerda con la representación «fotográfica» del sistema económico y permite hallar unos «precios de producción» indirectos o derivados de la «máquina» a edades diferentes. Puesto que son raros los mercados de bienes de capital fijo usados, tales precios no se pueden poner en correlación con precios efectivos, pero sí con valores contables, que también son fenómenos objetivos.

Desde el punto de vista crítico, los resultados más contundentes alcanzados por Sraffa se refieren a la imposibilidad de obtener una magnitud representativa de la «cantidad de capital» de una economía. Esto acarrea el descrédito de todos los modelos económicos que pretenden determinar de forma rigurosa el tipo de beneficios (o la equiparable tasa de interés) a partir de una magnitud objetiva («capital»). Sraffa demuestra que el capital no es una magnitud física, sino económica. Se puede calcular multiplicando un vector de cantidades (los medios de producción singulares) por un vector de precios. Pero los precios están condicionados por la distribución: cambios en la distribución modifican los precios y, por ende, el monto de capital. Por consiguiente, no es sostenible pretender que exista la «productividad marginal del capital». Como subrayó Joan Robinson en repetidas ocasiones, el capital puede ser concebido de dos formas: como un fondo de valor y como un conjunto heterogéneo de máquinas, edificios, materias primas. El capital sólo es socialmente productivo cuando el fondo de valor se transforma en medios de producción, que son objetos heterogéneos, destinados a funciones específicas y no transmutables unos en otros. 
Más aún, Sraffa demostró que podía muy bien ocurrir que dos técnicas no fueran ordenables en cuanto a su rentabilidad al margen del tipo de beneficios. En otras palabras, es perfectamente posible que la técnica A sea preferible a la técnica B para tipos de beneficio bajos, inferior para tipos de beneficio medianos y, de nuevo, superior para tipos de beneficio elevados. El descubrimiento de esta propiedad —considerada "paradójica» desde la óptica marginalista y conocida bajo el nombre de reswitching (readopción de técnicas)- refutaba los intentos de encontrar una forma «sustituta» de medición del capital que satisficiera la condición de que cuando el tipo de beneficios sube, las técnicas alternativas presentan necesariamente una «intensidad de capital» (o «relación capital por trabajador») decreciente.

Puesto que las consideraciones precedentes quizá aparezcan como un tanto abstrusas, será conveniente mostrar que los esquemas conceptuales criticados se hallan presentes y tienen un papel en las argumentaciones de historiadores de gran valía. Ha escrito Carlo M. Cipolla: «La herradura incrementó la eficacia del caballo y, por tanto, su valor. [...] El buey fue crecientemente sustituido por el caballo. [...] En esencia, la sustitución del buey por el caballo significó la sustitución de una forma de capital menos cara pero menos eficiente por otra más cara pero más eficiente" '. Desde un punto de vista sraffiano, se puede objetar que la primera proposición es lógicamente incorrecta ("por tanto») y revela precisamente una concepción del capital como un monto de valor que toma cuerpo en medios de producción. La argumentación contrapuesta seguiría los siguientes trazos: si el caballo herrado es un medio de producción más rentable que el buey, operará el desplazamiento de técnicas, no se repondrán bueyes y en pocos lustros desaparecerán, salvo que sean más útiles en procesos secundarios; ahora bien, los caballos son bienes reproducibles a través de procesos con rendimientos no decrecientes, por lo que su valor (o precio de producción) no tenderá a subir, aunque los precios efectivos se eleven durante la fase de sustitución de bueyes por caballos, merced al hecho de que la producción de éstos requiere tiempo. Por añadidura, el caballo pasaría a ser básico y el buey dejaría de serlo, con lo cual se sumaría un efecto difuso de abaratamiento de todos los bienes, incluido el caballo (valorados en términos de tiempo de trabajo incorporado).

\section{Enfoque}

Tras esta rápida ojeada a los resultados, puede ser esclarecedor orientar nuestra atención hacia el examen de las diferencias entre el enfoque de Sraffa y los enfoques dominantes en la teoría económica usual. Para caracterizar un

' Crpolla, Historia económica de la Europa preindustrial (Madrid, Alianza, 1981), pp. 175-176. 
enfoque, esto es, la manera de concebir y tratar cuestiones que se suscitan en cualquier campo de conocimiento, Mario Bunge propuso ${ }^{2}$ un esquema del siguiente tenor. Los rasgos determinantes de un enfoque son clasificables en cuatro bloques genéricos; a saber, andamiaje general, problemática, metódica y metas. Por andamiaje general se entiende un conjunto de hipótesis muy generales referentes al campo en cuestión, así como al modo de conocerlo; la problemática es el tipo de problemas que se desea tratar; la metódica, el conjunto de métodos o modos de tratar dichos problemas, y las metas, las finalidades últimas de la investigación de dichos problemas con dichos métodos. A pesar de tratarse de un planteamiento sumamente general, puede resultar útil para revelar el trasfondo de la obra de Sraffa. Conviene advertir, no obstante, que son muy escasas las referencias explícitas de nuestro autor sobre cuestiones epistemológicas, aunque es factible espigar una coherente colección de indicios.

El andamiaje general implícito en la obra de Sraffa puede ser descrito sintéticamente con dos atributos: realismo y sistemismo. El supuesto del realismo implica que la economía es considerada como una ciencia factual y que los datos de la experiencia no constituyen una instancia última, sino que tienen que explicarse a base de estructuras subyacentes sólo cognoscibles por vías indirectas, mediante procedimientos de contrastación complejos. En 1930, con motivo de un simposio sobre Rendimientos crecientes y empresa representativa, Sraffa manifestó: «Yo intento detectar los presupuestos implícitos en la teoría de Marshall; si Robertson los considera extremadamente irreales, simpatizo con él. Parece que estamos de acuerdo en que dicha teoría no puede ser interpretada de forma que tenga coherencia interna $y$, a la vez, esté de acuerdo con los hechos que quiere explicar. El remedio de Robertson consiste en descartar la matemática; tal vez yo debería haber expresado que mi opinión al respecto es que ha de descartarse la teoría de Marshall» ${ }^{3}$. Treinta años más tarde argumentaba contra Hicks en análogo sentido: "Con toda certeza la utilidad de cualquier teoría depende de su capacidad explicativa. [...] $\mathrm{Si}$ una teoría falla a la hora de explicar una situación, entonces es insatisfactoria» 4 .

${ }^{2}$ Cfr. Bunge, Epistemología (Barcelona, Ariel, 1880), p. 132.

${ }^{3}$ En el simposio participaron D. Robertson, G. Shove y P. Sraffa. Las intervenciones fueron publicadas bajo el título genérico "Increasing Returns and the Representative Firm - A Symposium", en el Economic Journal, XL, 1930, pp. 79116. El pasaje citado corresponde a Srarra, "Rejoinder", p. 93.

- Estas frases fueron pronunciadas en la Conferencia de Corfú sobre teoría del capital (4-11 de septiembre de 1958). Se encuentran citadas en el "Summary Record of the Debate", en Lutz y Hague (eds.), The Theory of Capital. Proceedings of a Conference held by the International Economic Association (Londres, Macmillan, 1961), p. 306. 
Por sistemismo queremos significar que las relaciones económicas son concebidas como propiedades sociales emergentes para cuya explicación no basta con referirse a los individuos. En concreto, por ejemplo, el valor económico para Sraffa se deriva esencialmente de las condiciones en que los hombres en sociedad, con un nivel técnico determinado y una particular estructura de clases, organizan la producción y reproducción de su vida material. Esto tiene poco que ver con las relaciones psíquicas entre sujetos y bienes, sobre las que se apoyan las teorías del valor subjetivo. En 1932, al reseñar un libro de Hayek, escribió nuestro autor: «El doctor Hayek, que ensalza los logros imaginarios del "método subjetivo" en economía, consigue a menudo poner de manifiesto cuán absurdo es" ${ }^{5}$.

La problemática que ocupa el lugar central en Producción de mercancias por medic de mercancias son los precios de producción, lo que revela una concepción objetivista del valor, según acabamos de ver. Asociada con ella se encuentra la cuantificación de las variables distributivas, que se hallan en un plano diferente a las variables precio, y la fijación de las relaciones que conectan todas esas variables. La caracterización de tales problemas deriva, con todo, de una visión subyacente que hace hincapié en que la mayoría de bienes son reproducibles y en que la actividad económica no se compone de procesos productivos y distributivos que transcurran enmarcados en una generalizada cooperación igualitaria, sino que se desenvuelven de forma jerarquizada y repetitiva.

En cambio, la problemática central del enfoque marginalista se refiere a la determinación de los precios y cantidades que aseguran el «equilibrio» del sistema. Para llegar a ese resultado se concentra la atención sobre el problema de la «escasez» y de las «elecciones» de los sujetos, fundamentalmente iguales, aunque poseedores de dotaciones diferentes, con un trasfondo de individualismo metodológico. Se desemboca así en una concepción del valor apoyada básicamente en la «utilidad» que los consumidores obtienen en el momento del cambio al anticipar su consumo efectivo. En tales enfoques de oferta/ demanda pueden coexistir elementos objetivos (costes) y subjetivos (funciones de utilidad) en un esquema híbrido, o puede dominar el elemento subjetivo si los costes se transmutan en «costes de oportunidad».

Desde un punto de vista global, por lo que atañe a los métodos, no hay contraposición genuina entre los dos enfoques. Ambos coinciden en la importancia de los métodos exactos. Pero mientras que en la teoría académica usual con frecuencia se sobreentiende que la corrección formal es un requisito suficiente, Sraffa subraya que el rigor debe extenderse al substrato conceptual y a las modalidades de contrastación (aunque a menudo sólo puedan ser indi-

s Srafra, "Dr. Hayek on Money and Capital", Economic Journal, XLII, 1932, página 47. 
rectas o indiciarias). Por eso, más que un repertorio de modelos autónomos y de difícil ensamblaje, Sraffa ha concedido un lugar preferente al método de las aproximaciones sucesivas como la forma más apropiada para aprehender una realidad sumamente compleja y en la que actúan elementos correspondientes a diferentes jerarquías y niveles.

En consonancia con lo que hemos ido viendo, la meta de Sraffa es alcanzar una visión representacional del sistema económico, por medio del descubrimiento de las relaciones fundamentales que caracterizan al objeto y dan razón de los mecanismos internos responsables decisivos de su comportamiento externo. Un alumno suyo lo ha relatado con las siguientes palabras: «Piero Sraffa me impresionó [en el período 1937-40] con su convicción de que era perfectamente posible, aunque difícil, transformar una teoría de la economía política en una ciencia exacta, basada en una precisión absoluta de conceptos - por difícil que fuera acercarnos a ello en el trabajo empíricoque pudiera manejarse con tanta eficacia como los instrumentos de un cirujano o de un soldador, para diseccionar o desmantelar, y luego volver a montar las interconexiones "invisibles" del proceso económico" ${ }^{6}$. Sin duda, también la economía académica usual se declara orientada hacia unas metas positivas o explicativas, pero mezcla estos objetivos con aspectos normativos y especulativos que dan lugar a modelos con estatuto equívoco. Quizá la limitación más llamativa es la adopción de postulados que resultan claramente irreales o contradichos por disciplinas vecinas (psicología, sociología, ecología), sin que ello sea motivo de preocupación expresa por parte de muchos teóricos. Eso da lugar a frecuentes acusaciones de irrelevancia contra las que, como antídoto, se enarbolan a menudo propiedades como la belleza, elegancia o el rigor formal, caracteres todos ellos poco decisivos para la evaluación del grado de verdad de una teoría.

\section{Srafta y la bistoria económica}

En Producción de mercancías por medio de mercancias no hay dinero, ni sindicatos, ni gobierno, ni demanda, ni monopolios, ni comercio exterior, ni elecciones, ni paro, ni inflación, ni referencia alguna a la historia externa de las ideas. En dicho libro, Sraffa sólo toma en consideración aquellos elementos necesarios para resolver su problema directo, la fijación de los precios de producción, y excluye todas las características que no son determinantes para la resolución de su problema. No obstante lo anterior, la tesis que deseo sugerir, como colofón de esta nota necrológica, es que Sraffa suministra una só-

- El testimonio pertenece a Arun Bose. Cfr. Bose, Economía politica marxiana $y$ postmarxiana (Madrid, Alianza, 1975), pp. 13-14 (traducción revisada). 
lida base para la historia económica, aunque hagan falta considerables esfuerzos de concreción y adaptación para pasar de la visión general a esquemas operativos. Esfuerzos indispensables tanto por el elevado nivel de abstracción en el que se sitúa aquel libro como por el hecho de que la economía se halla siempre combinada con elementos culturales, políticos y sociales que son resultado de procesos históricos previos.

Es evidente que los precios de producción, que constituyen la problemática explícita sobre la que discurre la investigación de Sraffa, se refieren a un contexto capitalista. Sin embargo, el enfoque sraffiano puede ser fácil. mente aplicable a otros sistemas económicos sin perder sus señas de identidad. Tres características clave posibilitan esa extensión; a saber, la visión reproductiva, la definición de excedente y el sello de temporalidad inherente a la forma en que Sraffa presenta los procesos de producción.

En efecto, es un hecho que la reproducción llevada a cabo mediante métodos de producción y de consumo productivo constituye un rasgo genérico propio de todos los períodos de la evolución humana, dado que traduce requerimientos biológicos de intercambio metabólico con la naturaleza. Es de destacar que la estabilidad de un sistema reproductivo concreto es condición suficiente para poder determinar «precios teóricos», hayan o no aparecido los precios como propiedades sociales emergentes. Nótese también que los precios de equilibrio de los marginalistas se contraponen a los precios de producción de los economistas clásicos y Sraffa, aunque tanto unos como otros aspiren a representar los precios efectivos. Ahora bien, los primeros son precios que igualan demandas y ofertas, mientras que los segundos se obtienen equiparando el tipo de beneficios (o tasa de ganancia) en todas las industrias. El entramado de causas subyacentes remite, en el primer caso, a la escasez $y$, en el segundo, a las condiciones de reproducibilidad.

La definición del excedente (o "producto neto») como un flujo periódico de bienes presenta, asimismo, atractivos obvios. Se trata de un utensilio conceptual que obliga a contemplar el sistema como totalidad y permite evitar la introducción de ciertos supuestos psicológicos estrafalarios. Pone de relieve que cuando los procesos' son interdependientes no es, generalmente, lícito afirmar que un sector es más o menos productivo que otro. En fin, la simple existencia de un excedente impone la necesidad de introducir variables distributivas, lo que puede resultar un correctivo útil ante ciertas tendencias que se inclinan por presentar una visión armoniosa no sólo del presente, sino también del pasado, postulando intercambios implícitos de equivalentes (entre señor feudal y siervo, por ejemplo).

Con respecto a la temporalidad, hay que decir de entrada que el enfoque de oferta y demanda constituye una extensión de la lógica de los intercambios a todo el campo económico. Comercio y producción presentan, sin em- 
bargo, notas diferenciadas, aunque formalmente puedan ser parcialmente equiparados. Ahora bien, mientras que para los intercambios el tiempo no constituye sino un telón de fondo pasivo, la producción es típicamente un proceso temporal. Así, los intentos de dinamizar las teorías del equilibrio no sólo no han conseguido resultados sustantivos, sino que requieren la introducción de supuestos que dan la impresión de bancarrota («hoy» se realizan todos los contratos para los $n$ períodos siguientes y, a veces, hasta para infinitos períodos sucesivos). El enfoque sraffiano, por el contrario, aparece como directamente temporalizado, aunque esta dimensión haya sido esterilizada (merced al supuesto de estado estacionario) a fin de abordar selectivamente otros problemas; pero la estructura básica no presenta signos de rechazo cuando se procede a injertar en ella elementos peculiares de la temporalidad.

\section{Orientación bibliográfica}

Vale la pena acudir directamente a la obra matriz, es decir, a SRAFfa, P.: Producción de mercancias por medio de mercancías, Barcelona, Oikos, 1966, dado que se trata de un texto breve y sin grandes complejidades técnicas. De todos modos, hay que recalcar que se trata de una obra difícil, aunque sea muy clara y aparentemente poco matemática.

El estudio más sistemático sobre el enfoque utilizado por Sraffa, su trayectoria intelectual y su ubicación en la historia de las ideas económicas es el libro de Roncaglia, A.: Sraffa y la teoría de los precios, Madrid, Pirámide, 1980.

Una buena colección de recensiones y comentarios a Producción de mercancias por medio de mercancias es la reunida y prologada por Caballero, A.: «Piero Sraffa y la "Producción de mercancías por medio de mercancías". Su significado en la teoría económica», Revista Española de Economía, V, 2 (mayo-agosto 1975), pp. 247-418, que recoge aportaciones de J. Robinson, R. F. Harrod, P. Sraffa, K. Bharadwaj, R. E. Quandt, G. C. Harcourt y V. G. Massaro, E. J. Nell, M. Dobb, J. Eatwell, P. K. Newman, E. Zaghini.

La mejor descripción genealógica de los problemas afrontados por Sraffa se encuentra en el libro de DовB, M.: Teoría del valor y de la distribución desde Adam Smith, Buenos Aires, Siglo XXI, 1975.

Un excelente texto específico sobre las dificultades que planean sobre la categoría «capital» en la economía marginalista, desde un punto de vista sraffiano, es Garegnani, P.: El capital en la teoria de la distribución, Barcelona, Oikos, 1982.

Un intento meritorio, pero no plenamente logrado, de traducir los planteamientos sraffianos (junto con los de Keynes y Kalecki) a manual introduc- 
torio fue llevado a cabo por Robinson, J., y EATwell, J.: Introducción a la economia moderna, Madrid, Fondo de Cultura Económica, 1976.

A nivel superior, con bastante aparato formal y la limitación de abarcar un territorio más reducido, destaca PAsinetri, L.: Lecciones de teoría de la producción, Madrid, Fondo de Cultura Económica, 1983.

No es aquí el lugar para repasar los intentos con vistas a desarrollar la investigación científica desde un enfoque sraffiano o similar. Señalemos, simplemente, que la revista en que tienen mayor cabida este tipo de inquietudes es Cambridge Journal of Economics, publicación trimestral nacida en 1977 y que expresamente considera como legado principal la obra de Marx, Kalecki y Keynes.

En áreas más próximas a la historia económica, merece ser anotado el esclarecedor intento de reinterpretar las doctrinas económicas utilizando esquemas sraffianos llevado a cabo por CARTELIER, J.: Excedente y reproducción, México, Fondo de Cultura Económica, 1981.

Un ensayo con vistas a compaginar marxismo y sraffianismo en el campo del estudio de los modos de producción se esboza en Barcelo, A.: Reproducción económica y modos de producción, Barcelona, Serbal, 1981.

Una excelente muestra de inteligente recepción para el campo de la antropología económica de las aportaciones de Sraffa se halla en Gudeman, S.: «Antropología económica: el problema de la distribución», in Llobera (comp.), Antropología económica (Barcelona, Anagrama, 1981), pp. 231-265.

Los intentos más prometedores de aplicación del enfoque sraffiano a la historia económica son los artículos de NELL, E. J., recogidos en un volumen que aparecerá en breve con el título (provisional): Historia y teoría económica, Barcelona, Crítica, 1984. 